BULL. AUSTRAL. MATH. SOC.

\title{
PERIODIC POINTS AND CHAOS FOR EXPANDING SELF-MAPS OF THE INTERVAL
}

\section{ZHANG ZHENHUA}

It is shown that expanding self-maps of the interval with a finite number of turning points must have periodic points whose periods are not integral power of 2 and therefore are chaotic.

\section{Introduction}

Let $I$ be the unit interval $[0,1]$ of the real line. A continuous map $f$ from $I$ to itself is piecewise monotonic if $I$ can be subdivided into finite number of subintervals $I_{1}, I_{2}, \ldots, I_{l}$ on which $f$ is either strictly increasing or strictly decreasing. Each such maximal interval on which $f$ is monotonic is called a lap of $f$, and $l=l(f)$ is the lap number of $f$. The separating points $c_{1}, c_{2}, \ldots, c_{l-1}$ at which $f$ has a local minimum or maximum are called the turning points of $f$. The limit $S(f)=\underset{n \rightarrow \infty}{\operatorname{limit}} l\left(f^{n}\right)^{1 / n}$ is a real number in the interval $[1, Z(f)]$ called the growth number of $f$. A piecewise monotonic map $f$ from $I$ to itself is expanding if there exists a constant $\lambda>1$ such that $|f(x)-f(y)| \geq \lambda|x-y|$ whenever both $x$ and $y$ belong to the same lap. Call $\lambda$ an expansion constant for $f$.

In recent years there has been considerable interest in the dynamical

Received 30 November 1984. The author wishes to thank his tutor Professor Chen Zaoping for his guidance,

Copyright Clearance Centre, Inc. Serial-fee code: 0004-9727/85 $\$$ A2. $00+0.00$. 
properties of difference equations defined by self-maps of the unit interval. The complicated asymptotic behaviour which often arises has been emphasized by the use of the term "chaotic" to characterize certain dynamical properties of a large class of such equations [1], [3], [5], [6], [8], [9]. This complexity can be dealt with statistically if the transformation admits an invariant measure [4] especially one which is absolutely continuous with respect to Lebesgue measure. Thus there has been much interest in proving the existence of such measures [7].

However, the connection between these two ideas has not yet been clarified. It is known that transformations with a periodic point whose period is not an integral power of 2 must exhibit chaotic behaviour [1], [3], [11]. On the other hand, if there exists some natural number $m$ such that the map $f^{m}$ is expanding then $f$ admits an absolutely continuous invariant measure [10]. In [2], Byers has shown that expanding maps with a unique turning point must have a periodic point of period $2^{n} \cdot 3$ and therefore are chaotic. In this paper we generalize the result and show that if there exists some natural number $m$ such that the map $f^{m}$ is expanding then $f$ admits a periodic point whose period is not an integral power of 2 and therefore is chaotic.

\section{Discussion}

LEMMA 1. If $f: I \rightarrow I$ is a continuous expanding map with expansion constant $\lambda$ then, for any natural number $n, f^{n}$ is an expanding map with expansion constant $\lambda^{n}$.

Proof. Let $c_{1}, c_{2}, \ldots, c_{l-1}$ be the turning points of $f$. Set

$$
E=\bigcup_{j=0}^{n-1}\left(\bigcup_{i=1}^{l-1} f^{-j}\left(G_{i}\right)\right)
$$

We shall first show that $E$ is exactly the set of all turning points of $f^{n}$. For any $x_{0} \in E$, there must exist $1 \leq i_{0} \leq 2-1,0 \leq j_{0} \leq n-1$, such that $f^{j_{0}}\left(x_{0}\right)=c_{i_{0}}$. Hence, for any natural number $j \geq j_{0}+1, f^{j}$ 
has a local extreme value at $x_{0}$, in particular, so does $f^{n}$. Thus $x_{0}$ is a turning point of $f^{n}$. Now let us assume that $x^{*}$ is a turning point of $f^{n}$. If $x^{*} \neq E$, then, for any $j$ which satisfies the condition that $0 \leq j \leq n-1, f^{j}\left(x^{*}\right)$ is not a turning point of $f$; that is $f$ is monotonic at $f^{j}\left(x^{*}\right)$, in particular at $f^{n-1}\left(x^{*}\right)$. Hence $f^{n}$ is monotonic at $x^{*}$ and this contradicts the assumption that $x^{*}$ is a turning point of $f^{n}$. Therefore $x^{*} \in E$.

Finally we shall show that $f^{n}$ is an expanding map with expansion constant $\lambda^{n}$. Let us take an arbitrary lap $I_{k}^{(n)}$ of $f^{n}$. For any $x$ and $y$ belonging to $I_{k}^{(n)}$, we may assume that $x<y$ without loss of generality. Then for each fixed $j=0,1, \ldots, n-1, f^{j}(x)$ and $f^{j}(y)$ belong to the same lap of $f$. Otherwise from the continuity of $f$ there must exist $x_{0}$ which belongs to $(x, y)$ and $c_{i_{0}}$ such that $f^{j}\left(x_{0}\right)=c_{i_{0}}$, that is, $x_{0} \in E$ and this contradicts the assumption that $x, y \in I_{k}^{(n)}$. Therefore

$$
\left|f^{n}(x)-f^{n}(y)\right| \geq \lambda\left|f^{n-1}(x)-f^{n-1}(y)\right| \geq \ldots \geq \lambda^{n}|x-y| .
$$

That is $\left|f^{n}(x)-f^{n}(y)\right| \geq \lambda^{n}|x-y|$.

LEMMA 2 [10]. Suppose the continuous map $f: I \rightarrow I$ is piecewrise monotonic. If $S(f)>1$, then $f$ admits a periodic point whose period is not an integral power of 2 .

THEOREM. If $f: I \rightarrow I$ is a continuous expanding map with a finite number of turning points, then $f$ admits a periodic point whose period is not an integral power of 2 and therefore is chaotic.

Proof. For an arbitrary natural number $n$, we may take any lap $I_{k}^{(n)}=\left[c_{k-1}^{(n)}, c_{k}^{(n)}\right]$ of $f^{n}$. Let $\lambda$ be an expansion constant of $f$, then we have $\left|f^{n}\left(c_{k}^{(n)}\right)-f^{n}\left(c_{k-1}^{(n)}\right)\right| \geq \lambda^{n}\left|c_{k}^{(n)}-c_{k-1}^{(n)}\right|$ by Lemma 1. Hence 
$\left|c_{k}^{(n)}-c_{k-1}^{(n)}\right| \leq \lambda^{-n}$ since $\left|f^{n}\left(c_{k}^{(n)}\right)-f^{n}\left(c_{k-1}^{(n)}\right)\right| \leq 1$, that is, the length of $I_{k}^{(n)}$ is equal to or less than $\lambda^{-n}$. Thus the lap number $2\left(f^{n}\right)$ of $f^{n}$ is equal to or larger than $1 / \lambda^{-n}=\lambda^{n}$, that is $l\left(f^{n}\right)^{1 / n} \geq \lambda$; hence $S(f) \geq \lambda>1$. By Lemma $2, f$ admits a periodic point whose period is not an integral power of 2 and therefore is chaotic.

COROLLARY. Suppose the continuous map $f: I \rightarrow I$ is piecewise monotonic. If there exists a natural number $m$ such that $f^{m}$ is expanding, then $f$ admits a periodic point whose period is not an integral power of 2 and therefore is chaotic.

Proof. Let $g=f^{m}$. Then $g$ satisfies the conditions of the theorem; hence $g$ admits a periodic point whose period is not an integral power of 2 . So $f$ possesses a periodic point of period not equal to $2^{n}$ for any natural number $n$ and therefore is chaotic.

We give the following example to illustrate the corollary.

EXAMPLE. $f: I \rightarrow I$ is defined in the following way:

$$
f(x)= \begin{cases}-3 x / 2+1, & x \in[0,2 / 3], \\ 3 x / 4-1 / 2, & x \in[2 / 3,1] .\end{cases}
$$

Hence

$$
f^{2}(x)= \begin{cases}-9 x / 8+1 / 4, & x \in[0,2 / 9] \\ 9 x / 4-1 / 2, & x \in[2 / 9,2 / 3] \\ -9 x / 8+7 / 4, & x \in[2 / 3,1]\end{cases}
$$

It is obvious that $f$ is not expanding, but $f^{2}$ is an expanding map.

\section{References}

[1] G.J. Butler and G. Pianigiani, "Periodic points and chaotic functions in the unit interval", Bull. Austral. Math. Soc. 18 (1978), $255-265$. 
[2] Bill Byers, "Periodic points and chaos for expanding maps of the interval", Bull. Austral. Math. Soc. 24 (1981), 79-83.

[3] Frederick J. Fugl ister, "A note on chaos", J. Combin. Theory Ser. A 26 (1979), 186-188.

[4] J. Guckenheimer, G. Oster and A. Ipaktchi, "The dynamics of density dependent population models", J. Math. Biol. 4 (1977), 101-147.

[5] James L. Kaplan and Frederick R. Marotto, "Chaotic behaviour in dynamical systems", Nonlinear systems and applications, 199-210 (Proc. Internat. Conf. Nonlinear Systems and Applications, University of Texas, Arlington, July 1976. Academic Press [Harcourt Brace Jovanovich], New York, San Francisco, London, 1977).

[6] Peter E. Kloeden, "Chaotic difference equations are dense", Bull. Austraz. Math. Soc. 15 (1976), 371-379.

[7] A. Lasota and James A. Yorke, "On the existence of invariant measures for piecewise monotonic transformations", Trans. Amer. Math. Soc. 186 (1973), 481-488.

[8] Tien-Yien Li and James A. Yorke, "Period three implies chaos", Amer. Math. Monthly 82 (1975), 985-992.

[9] Tien-Yien Li, M. Misiurewicyz, G. Pianigiani and James A. Yorke, "Odd chaos", Phys. Lett. A 87 (1982), 271-273.

[10] J. Milnor and W. Thurston, "On iterated maps of the interval", preprint.

[11] Y. Dono, "Period $\neq 2^{n}$ implies chaos", Progr. Theoret. Phys. 59 (1978), 1028-1030.

\author{
Department of Mathematics, \\ Hebei Teachers' University, \\ Shijiazhuang, \\ Hebei Province, \\ The People's Republic of China.
}

